\title{
Association of oral microbiota with obesity in children: insight from dental physicians
}

Hong Kong Med J 2020;26:354

https://doi.org/10.12809/hkmj208479

To the Editor-Oral microbiota composition varies in normal individuals from birth until adulthood because of various intrinsic and extrinsic factors. Initially Gram-positive aerobic microbial species (streptococcus variants) grow with varying degrees of increase in facultative and strict Gram-negative anaerobic microbial species as age advances and with alteration of periodontal status. Obesity in children is a major risk factor for future cardiovascular diseases, diabetes, gastrointestinal disorders, and dental diseases. ${ }^{1}$ Faecal microbiota have been identified as causes of central obesity. The gut is home to trillions of microbes-about 10 times more than the number of human cells-despite the strong action of acids from the stomach and small and large intestines. A definite association of increased abundance of Firmicutes and lack of Bacteroides spp is related to central obesity. However, the association between oral microbiota and obesity has yet to be investigated.

Gram-negative bacteria such as Porphyromonas gingivalis, Tannerella forsythia, Proteobacteria spp, Campylobacter rectus, Neisseria mucosa, and Selenomonas noxia have been detected in the subgingival film of obese individuals, and a four- to six-fold increase in Proteobacteria spp, $C$ rectus, and $N$ mucosa has been reported in obese patients. ${ }^{2}$ However these associations in children remain unclear. Circulating adipokines might influence the immune response at the mucosal level in the oral cavity, thereby affecting the microbial colonisation. Also, at the cellular level, macrophages may produce a number of pro-inflammatory cytokines, interleukin-1, and tumour necrosis and prostaglandins that contribute to chronic inflammation and physiopathological mechanisms involved in the development of obesity. ${ }^{3}$

Little is known about the oral microbiota in children. As dental physicians, our understanding regarding the relationship between oral health and childhood growth could help identify preventable factors contributing to obesity and related conditions, including onset of menarche which is associated with obesity. ${ }^{4}$ Further studies are required to clarify the effects of growth of specific oral microbiota with growth patterns. Multidisciplinary research including dental surgeons and general physicians to identify the association of oral microbiota with obesity in children may prevent future major cardiovascular diseases.

\section{Author contributions}

The authors contributed to the letter, approved the final version for publication, and take responsibility for its accuracy and integrity.

\section{Conflicts of interest}

All authors have disclosed no conflicts of interest.

\section{Funding/support}

This letter received no specific grant from any funding agency in the public, commercial, or not-for-profit sectors.

Vaishnavi Vedam *, MDS (Oral Pathology \& Microbiology) Sivadas Ganapathy, MDS (Paediatric Dentistry)

Faculty of Dentistry, Asian Institute of Medicine, Science and Technology (AIMST) University, Malaysia

*Corresponding author: vaishnavivedam@gmail.com

\section{References}

1. Ulloa PC, van der Veen MH, Krom BP. Review: modulation of the oral microbiome by the host to promote ecological balance. Odontology 2019;107:437-48.

2. Craig SJ, Blankenberg D, Parodi AC, et al. Child weight gain trajectories linked to oral microbiota composition. Sci Rep 2018;8:14030.

3. Zeigler CC, Persson GR, Wondimu B, Marcus C, Sobko T, Modéer T. Microbiota in the oral subgingival biofilm is associated with obesity in adolescence. Obesity (Silver Spring) 2012;20:157-64.

4. Mervish NA, Hu J, Hagan LA, et al. Associations of the oral microbiota with obesity and menarche in inner city girls. J Child Obes 2019;4:2. 